\title{
Éditorial
}

\section{Enjeux dans la Vallée}

Zone économique désignée en 2002 pour une région productrice d'aluminium depuis 1926, la «Vallée de l'aluminium » tente d'appliquer un concept pertinent. La théorie économique offre en effet à la Vallée un ancrage scientifique déjà ancien sous la forme du « district industriel ». Concept classique qui fut largement renouvelé récemment à la lumière des faits $^{1}$ avec l'analyse des NDI (nouveaux districts industriels), des milieux innovateurs, des systèmes territoriaux d'innovation, des grappes industrielles, des communautés intelligentes et autres learning regions. Ainsi, la Vallée de l'aluminium bénéficie d'un riche corpus scientifique qui permet de justifier son existence et d'inspirer des actions pour son organisation territoriale.

Ce numéro thématique de la Revue de vulgarisation scientifique O\&T ne vise pas un avancement théorique. Son objectif concerne plutôt un jalon d'analyse territoriale de la grappe industrielle du secteur de l'aluminium, localisée dans une vallée nordique du Québec. D'emblée, il est pertinent de soulever cinq enjeux importants auxquels fait face la Vallée de l'aluminium dans son désir de mise en œuvre du concept de district industriel ${ }^{2}$. Il s'agit de la territorialité, du déséquilibre valeur - bénéfices, de la stratégie industrielle, de la comptabilité du phénomène et de l'apprentissage collectif.

En matière de territorialité, le découpage régional Saguenay-Lac-Saint-Jean retenu pour asseoir la Vallée ne correspond ni à la concentration de la grande majorité des activités économiques au sein du corridor urbain Alma - La Baie, et non plus à la dispersion de celles-ci au-delà de la région, notamment à Baie-Comeau, Sept-Îles, Shawinigan. Les acteurs étant sensibles aux effets de proximité mais aussi relativement mobiles dans l'espace, ce flou territorial ajoute ainsi à la difficulté d'imposer l'image référentielle de la Vallée de l'aluminium à une région qui s'identifie par ailleurs à bien d'autres symboles $^{3}$ forts tels que le mythe du Royaume, l'oasis nordique, le Laboratoire de développement durable, le Domaine-du-Roy, la pépinière d'artistes, la Boréalie, la Sagamie, les Bleuets. Bref, la Vallée qui s'offre vigoureusement comme référent territorial respecte mal la territorialité de la grappe industrielle en question et n'apporte aucune solution globale à la symbolique identitaire tout en confirmant sa fragmentation peu propice à la synergie.

À cet effet, signalons que l'industrie de l'aluminium localisée dans ces lieux nordiques s'avère tributaire de l'énergie hydroélectrique produite dans l'arrièrepays. Pays «d'où l'eau jaillit » selon la toponymie des Premières Nations ${ }^{4}$. Pays qui contient des territoires ancestraux amérindiens, de nombreux sites récréotouristiques, des bassins miniers, des terres fertiles, une boréalie comme puits de carbone, un potentiel humain de créativité remarquable, et aussi d'immenses gisements d'eau qui peuvent représenter en l'occurrence, une image symbolique transcendante pour un éventuel positionnement mondial intégré de ses divers attributs territoriaux.

Le $2^{\text {ème }}$ enjeu opérationnel de la Vallée de l'aluminium réside dans le rapport inégal entre la valeur d'usage industriel tirée de l'hydroélectricité turbinée à faibles coûts de revient et les bénéfices induits qui profitent réellement à la région productrice. D'une part, la rente hydroélectrique s'accroît constamment pour Rio Tinto - Alcan, au fil de la tendance nette à la hausse des prix mondiaux de l'énergie payés par l'industrie ${ }^{5}$. D'autre part, le nombre de postes de travail nécessités pour la fonte d'aluminium décroît fortement dans la Vallée ${ }^{6}$, entraînant des conséquences économiques et sociales considérables. Ces deux tendances divergentes accentuent le déséquilibre initial dans la distribution de la rente hydroélectrique. Distribution pour laquelle un consensus régional a été possible jadis afin d'assurer la profitabilité d'une corporation canadienne ainsi que l'industrialisation régionale. Mais ce consensus devient insoutenable actuellement dans une région en déclin ${ }^{7}$, désappropriée de sa principale richesse en gains de valeur pour la corporation monopolistique ${ }^{8}$ devenue australienne. Tôt ou tard la société civile du Saguenay_Lac-SaintJean imposera la recherche d'une solution à ce déséquilibre croissant.

Pour pallier aux quelque 10000 emplois directs et indirects perdus en trois décennies grâce aux gains de productivité du complexe industriel de l'aluminium au Saguenay-Lac-Saint-Jean, la stratégie régionale de compensation qui fut retenue par le milieu ${ }^{9}$ concerne la transformation de l'aluminium. Après vingt-cinq ans de soutien public à cette stratégie, les effets réels sur la structure industrielle de la Vallée demeurent modestes malgré certains succès qui apparaissent exceptionnels dans un environnement relativement contraignant. Or, les vénérables ALUTECH, DEVINCI et autres Novelis qui génèrent autour de 800 emplois ${ }^{10}$ dans la transformation de l'aluminium, ne peuvent faire oublier que la Vallée 
nécessite impérativement de nouvelles options stratégiques. S'inscrit en ce sens l'attention générale très actuelle focalisée sur le segment des équipementiers. Focus qui fut imposé par Rio Tinto Alcan afin d'une part de gommer son retrait de la stratégie régionale de transformation du métal et, d'autre part, de socialiser certains coûts de l'innovation dans les équipements nécessaires à l'implantation de sa nouvelle technologie AP-50. Habilement claironnée, cette option certes intéressante pour certaines entreprises n'est aucunement suffisante pour la structuration industrielle. Le problème de la transformation de l'aluminium demeure entier dans la Vallée.

Le quatrième enjeu associé à la Vallée réside dans la difficile comptabilité territoriale applicable à un tel district industriel en émergence qui n'épouse pas une rubrique officielle de Statistiques Canada. Or, la Vallée bénéficie heureusement d'efforts divers de compilations de données, peu coordonnés cependant, mais néanmoins pertinents vers la mise en place d'une base empirique. Malgré les acquis indéniables en ces sens, nous connaissons encore mal les principales composantes de la Vallée et encore moins celles de l'industrie mondiale de l'aluminium en pleine croissance, notamment les stratégies utilisées ici et là sur la planète pour maximiser les retombées territoriales. À notre avis, un laboratoire de recherche économique sur l'aluminium devient essentiel dans la Vallée.

Question centrale de la théorie des districts industriels, l'apprentissage collectif à l'innovation qui est en principe favorisé par la proximité territoriale des acteurs, représente le dernier enjeu soulevé ici à propos de la Vallée de l'aluminium. Dépendant de diverses conditions institutionnelles, cet apprentissage collectif est généralement associé à la qualité de l'interaction. Or selon notre lecture comparative, l'interaction apparaît suffisamment dense dans la Vallée, notamment depuis que le « réseautage » est devenu une pratique systématique qui s'ajoute aux regroupements traditionnels. Ainsi, de petits événements d'interaction ciblés se multiplient, sous diverses initiatives. Alors que certaines «têtes de réseaux » illustrent leurs vertus de catalyse, notamment la Société de la Vallée de l'aluminium et le Comité de la Carte routière technologique. Selon notre observation encore imparfaite de cette interaction ${ }^{11}$, certaines contraintes limitent l'apprentissage collectif générateur d'innovations sous ses diverses formes. Signalons en particulier la faible et très faible connectivité de certains acteurs pourtant importants dans la Vallée. En outre, les replis exacerbés sur certains réseaux-égo et certains réseaux-clique contraignent la mise en œuvre naturelle d'un « réseau de réseaux » horizontal et transitif, capable en principe de doter la Vallée d'une vision globale partagée, réflexive et constamment renouvelée ainsi que d'une mission articulée et engageante à l'égard de l'apprentissage collectif, de la créativité et de l'innovation. Une solution devient nécessaire. Elle réside à notre avis dans la mise en œuvre d'un mécanisme institutionnel dans le style «Forum permanent» afin de collecter, traiter, cumuler, fertiliser et faire circuler l'information de nature cognitive.

Le concept opérationnel de Vallée de l'aluminium a déjà fait couler beaucoup d'encre au Québec et ailleurs. Avec ce modeste numéro thématique, la revue Organisations et Territoires apporte une contribution supplémentaire vers une meilleure intelligence collective des enjeux, des problèmes et des solutions. Nous espérons que ce cumul de connaissances ainsi stimulé sur le sujet fasse boulede-neige.

Bonne lecture.

Marc-Urbain Proulx

Le Directeur

Voir Proulx, M.-U. (2002) «L'Économie des territoires au Québec », P.U.Q., Québec, 364 p.

Il s'agit en réalité du $2^{\text {ème }}$ effort systématique pour mettre en place un district industriel au Saguenay puisque cette ambition explicite bien documentée par les historiens motiva grandement J.E.A. Dubuc, J.D. Guay et autres « Américains du Saguenay » au début du XXème siècle dans leurs efforts systématiques (finance, transport, information, marketing, formation, démarchage...) à l'égard de l'industrialisation de cette zone.

3 Voir Girard, C. et Perron, N. (1996) « Histoire du Saguenay-Lac-Saint-Jean », P.U.L. Québec, 665p.

4 Voir l'article de Mgr Victor Tremblay (2005) «Le nom de Saguenay dans l'histoire », dans Revue Organisations et Territoires, vol 14, no 2, pp.19-26. Original publié dans Saguenayensia, vol 5, no 5-6, 1963

5 Selon le CRU (Commodity Research Unit) de Londres, le prix moyen payé par l'industrie de l'aluminium s'est accrû de $44 \%$ entre 2003 et 2007. Bondissement ponctuel exceptionnel, certes, qui se situe néanmoins dans une tendance lourde à la hausse mondiale de la valeur de l'énergie et à la rareté des lots offerts aux alumineries.

6 Depuis 1980, la corporation Rio Tinto - Alcan a soustrait 3850 postes de travail (emplois directs), soit 39\% des emplois industriels générés par son complexe régional de production, malgré l'implantation de trois nouvelles alumineries intenses en technologies (La Baie - Laterrière - Alma) qui lui ont permis de doubler sa production d'aluminium primaire pour atteindre près d'un million de tonnes métriques.

Voir Proulx, M.-U. (2007) « Vision 2025 : le Saguenay—Lac-Saint-Jean face à son avenir », P.U.Q., Québec, 263 p.

Voir l'article de Diallo, T. dans ce numéro de la revue Organisations et Territoires

Voir les deux numéros spéciaux de la Revue Organisations et Territoires portant sur le Saguenay—Lac-Saint-Jean, soit vol. 13, no 2 (2004) et vol. 14, no 2 (2005).

10 Selon nos mesures, ces emplois ne représentent que $9 \%$ de la $1^{\text {ère }}$ transformation et seulement $4 \%$ de la $2^{\text {ème }}$ transformation, au Québec.

11 Voir l'article sur « L'interaction dans la Vallée » dans ce numéro de la revue Organisations et Territoires. 